\title{
Coulomb drag in coherent mesoscopic systems
}

\author{
Mortensen, Asger; Flensberg, Karsten; Jauho, Antti-Pekka
}

Published in:

Physical Review Letters

Link to article, DOI:

10.1103/PhysRevLett.86.1841

Publication date:

2001

Document Version

Publisher's PDF, also known as Version of record

Link back to DTU Orbit

Citation (APA):

Mortensen, A., Flensberg, K., \& Jauho, A-P. (2001). Coulomb drag in coherent mesoscopic systems. Physical Review Letters, 86(9), 1841-1844. https://doi.org/10.1103/PhysRevLett.86.1841

\section{General rights}

Copyright and moral rights for the publications made accessible in the public portal are retained by the authors and/or other copyright owners and it is a condition of accessing publications that users recognise and abide by the legal requirements associated with these rights.

- Users may download and print one copy of any publication from the public portal for the purpose of private study or research.

- You may not further distribute the material or use it for any profit-making activity or commercial gain

- You may freely distribute the URL identifying the publication in the public portal

If you believe that this document breaches copyright please contact us providing details, and we will remove access to the work immediately and investigate your claim 


\title{
Coulomb Drag in Coherent Mesoscopic Systems
}

\author{
Niels Asger Mortensen, ${ }^{1,2}$ Karsten Flensberg, ${ }^{2}$ and Antti-Pekka Jauho ${ }^{1}$ \\ ${ }^{1}$ Mikroelektronik Centret, Technical University of Denmark, Ørsteds Plads 345 east, DK-2800 Kgs. Lyngby, Denmark \\ ${ }^{2} \emptyset$ rsted Laboratory, Niels Bohr Institute, Universitetsparken 5, DK-2100 Copenhagen Ø, Denmark
}

(Received 5 July 2000)

\begin{abstract}
We present a theory for Coulomb drag between two mesoscopic systems. Our formalism expresses the drag in terms of scattering matrices and wave functions, and its range of validity covers both ballistic and disordered systems. The consequences can be worked out either by analytic means, such as the random matrix theory, or by numerical simulations. We show that Coulomb drag is sensitive to localized states, which usual transport measurements do not probe. For chaotic $2 \mathrm{D}$ systems we find a vanishing average drag, with a nonzero variance. Disordered 1D wires show a finite drag, with a large variance, giving rise to a possible sign change of the induced current.
\end{abstract}

PACS numbers: 73.23.-b, 73.50.-h, 73.61.-r

Moving charges in a conductor exert a Coulomb force on the charge carriers in a nearby conductor, thus inducing a drag current (see Fig. 1). This happens whenever the distance between the two conductors is of the same order as the average distance between charge carriers. In recent years Coulomb drag in two-dimensional systems has been studied extensively [1] and has provided valuable information about the interactions between adjacent extended electron gases.

Coulomb drag of mesoscopic structures has been addressed in the case of 1D systems both within the Boltzmann equation approach [2] and for Luttinger liquids with strong interwire interactions [3].

The study of fluctuations in the mesoscopic regime was recently initiated by Narozhny and Aleiner [4], and it was established that fluctuations will dominate at temperatures smaller than the Thouless energy. This was predicted to be the case even for large extended samples, such as those used in the 2D experiments [1]. While Ref. [4] concentrated on structures larger than the phase-breaking length, $\ell_{\phi}$, here we study Coulomb drag of mesoscopic samples smaller than $\ell_{\phi}$. Experimentally there is so far only little work on drag in structures with $L<\ell_{\phi}$ [5]. We believe this would be an extremely promising new direction for the study of mesoscopic transport properties, since it gives an opportunity to directly study interaction and correlation effects in mesoscopic structures. Especially disordered mesoscopic systems are known to exhibit interesting and unusual physics, and the same can be expected for disordered Coulomb drag systems - perhaps even more so because Coulomb drag in addition to the dependence on the transmission properties also has a strong dependence on the nature of the wave function inside the mesoscopic region. We note that Coulomb coupling also has interest in other contexts, such as capacitive coupling of a mesoscopic conductor to the environment, charge pumping in quantum dots, or spin polarized transport [6].

Before presenting the technical details we state our main results. We develop a formalism for studying drag in mesoscopic systems, and apply it to a number of special cases.
In the case of 1D wires we find that even a small amount of disorder induces fluctuations, such that the drag can exceed the ballistic limit, be strongly suppressed, or even change sign. The sign change is a general feature of mesoscopic drag, which we also demonstrate for chaotic systems. Here arguments based on random matrix theory show that the drag is zero on average, while the fluctuations are finite. The zero average drag can thus be taken as a test of the degree of ergodicity of the system under investigation. Furthermore, we address the importance of localized states in the sample [7]. While localized states do not usually affect the ordinary transport properties, they turn out to be important for the transconductance. The reason is that the electron-electron interaction allows for transitions in and out of the localized states, which become visible at temperatures smaller than the level spacing, giving rise to peaks in the transconductance when they cross the Fermi level. We also find a temperature dependence which is very different from the $T^{2}$ dependence found for extended states.

General formulation.-Using linear response theory similar to Refs. [8,9] we find the Coulomb drag to second order in the interaction between mesoscopic subsystems, $U_{12}$, taking the isolated systems to be otherwise noninteracting. The general formula for the dc transconductance in the case of two mesoscopic conductors, as illustrated in

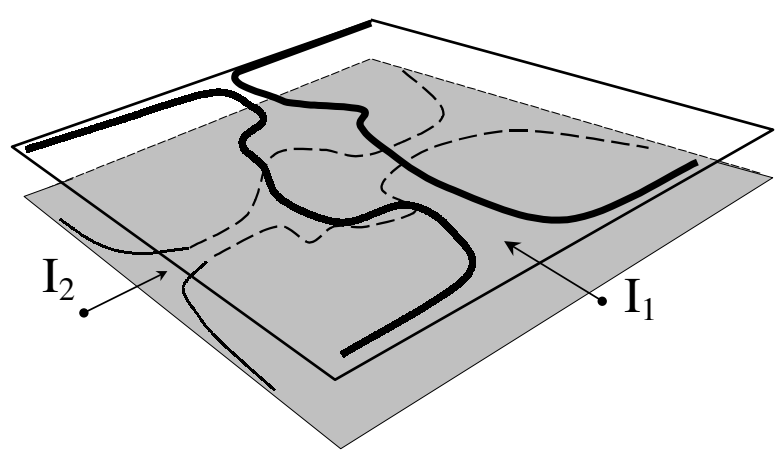

FIG. 1. Schematic geometry of a mesoscopic Coulomb drag experiment [15]. 
Fig. 1, is given by

$$
\begin{aligned}
G_{21}= & \frac{e^{2}}{h} \int d \mathbf{r}_{1} d \mathbf{r}_{2} d \mathbf{r}_{1}^{\prime} d \mathbf{r}_{2}^{\prime} U_{12}\left(\mathbf{r}_{1}, \mathbf{r}_{2}\right) U_{12}\left(\mathbf{r}_{1}^{\prime}, \mathbf{r}_{2}^{\prime}\right) \\
& \times \hbar \int_{-\infty}^{\infty} d \omega \frac{\Delta_{1}\left(\omega, \mathbf{r}_{1}, \mathbf{r}_{1}^{\prime}\right) \Delta_{2}\left(-\omega, \mathbf{r}_{2}, \mathbf{r}_{2}^{\prime}\right)}{2 k T \sinh ^{2}(\hbar \omega / 2 k T)}
\end{aligned}
$$

where $\Delta$ is the three point correlation function $\langle\hat{I} \hat{\rho} \hat{\rho}\rangle$, as explained in Ref. [9]. Equation (1) generalizes the results of Refs. [8,9] to systems with broken translation invariance. For the case of mesoscopic conductors it becomes

$$
\begin{aligned}
\Delta_{i}\left(\omega, \mathbf{r}, \mathbf{r}^{\prime}\right)= & -2 i \pi^{2} \hbar \sum_{\beta} \theta_{\beta}^{i}\left(\mathbf{r}, \mathbf{r}^{\prime}, \varepsilon_{\beta}-\hbar \omega\right) \\
& \times\left[n_{F}\left(\varepsilon_{\beta}-\hbar \omega\right)-n_{F}\left(\varepsilon_{\beta}\right)\right] \\
& +\left(\mathbf{r} \leftrightarrow \mathbf{r}^{\prime} ; \omega \rightarrow-\omega\right) .
\end{aligned}
$$

Here

$$
\theta_{\beta}^{i}\left(\mathbf{r}, \mathbf{r}^{\prime}, \varepsilon\right)=\sum_{\alpha \gamma} I_{\alpha \gamma}^{i} \rho_{\alpha \beta}^{i}(\mathbf{r}) \rho_{\beta \gamma}^{i}\left(\mathbf{r}^{\prime}\right) \delta\left(\xi_{\alpha}\right) \delta\left(\xi_{\gamma}\right),
$$

where $\xi_{\alpha}=\varepsilon_{\alpha}-\varepsilon$ and $i$ labels the subsystem. The matrix elements are given by $I_{\alpha \gamma}^{i}=\left\langle\alpha\left|\hat{I}^{i}\right| \gamma\right\rangle$ and $\rho_{\alpha \beta}^{i}(\mathbf{r})=$ $\langle\alpha \mid \mathbf{r}\rangle\langle\mathbf{r} \mid \beta\rangle$, where $|\alpha\rangle$ 's are the eigenstates of the uncoupled subsystem with energies $\varepsilon_{\alpha}$. Using scattering states as the basis we get $I_{\alpha \beta}^{i}=\frac{\hbar}{2 m} \delta_{\varepsilon_{\alpha}, \varepsilon_{\beta}} j_{\alpha \beta}$, where the matrix $j$ can be expressed in terms of the $2 N \times 2 N$ scattering matrix $S$ [10] as $j=\left(\tau^{3}-S^{\dagger} \tau^{3} S\right)$. Here $\tau_{n n^{\prime}}^{3}=$ $\pm \delta_{n n^{\prime}}$ with plus for $n$ belonging to right moving scattering states and minus for the left moving states.

Some general features immediately follow from Eq. (1). The usual cancellation of velocity and density of states, which is central in the derivation of the Landauer-Büttiker formula, occurs only for $I_{\alpha \gamma}^{i}$, whereas for $\rho_{\alpha \beta}^{i}$ this is not the case. Consequently, in contrast to individual subsystem conductances $G_{i i}, G_{21}$ peaks at the onset of new modes in either of the subsystems. Second, we notice that the sum over $|\beta\rangle$ mixes both propagating and evanescent modes. This means that apart from the transmission properties also localized states are probed by measuring drag conductance. Finally, we notice that the outcome of Eq. (1) can have any sign, which is directly related to lack of translation invariance.

The low temperature limit also follows readily from Eq. (1). The factor $\sinh ^{-2}$ cuts off the frequency integration, and we can expand the $\Delta$ 's to lowest order in $\omega$. This gives $\Delta \propto \omega$ with the sum over states restricted to those at the Fermi level $\left(\xi_{\beta}^{F}=\varepsilon_{\beta}-\varepsilon_{F}\right)$ :

$$
\Delta_{i}\left(\omega, \mathbf{r}, \mathbf{r}^{\prime}\right)=4 \omega \pi^{2} \hbar^{2} \operatorname{Im} \sum_{\beta} \theta_{\beta}\left(\mathbf{r}, \mathbf{r}^{\prime}, \varepsilon_{F}\right) \delta\left(\xi_{\beta}^{F}\right) .
$$

We immediately see that the transconductance in this limit becomes proportional to $T^{2}$, in accordance with the usual
Fermi liquid result for electron-electron scattering. Note, however, that the low temperature expansion breaks down when the temperature becomes smaller than the level spacing of the discrete, i.e., localized states, which we discuss in detail below. At higher temperatures the $T^{2}$ behavior is replaced by a weaker temperature dependence (e.g., for a quasi-1D system, $G_{21} \propto T$ for $k T>\hbar v_{F} / L$ as considered in Ref. [2]). Here we concentrate, however, on the low temperature dependence.

One-dimensional wires. - Next we consider as an illustrative example two disordered 1D wires, which we solve both numerically and analytically using perturbation theory. The one-dimensional case shows that a small amount of disorder can lead to large fluctuations for the drag response and even reverse the sign. The reason for this is that interwire interaction induced forward scattering gives rise to a drag response provided it is combined with disorder induced backscattering. In contrast, in the case of clean wires the backscattering is induced solely by the interwire interaction, and therefore the disordered case is larger by a factor of order $\langle\mathcal{R}\rangle U_{12}(0) / U_{12}\left(2 k_{F}\right)$, with $U_{12}(q)=$ $\int_{0}^{L} \int_{0}^{L} d x_{1} d x_{2} e^{i q\left(x_{1}-x_{2}\right)} U_{12}\left(x_{1}, x_{2}\right)$ being the Fourier transformed interaction and $\mathcal{R}$ the reflection coefficient, which is inversely proportional to the mean free path $\langle\mathcal{R}\rangle \simeq L / \ell$. We can show this explicitly by considering the lowest order perturbation theory in disorder potential, corresponding to the diagrams shown in Fig. 2, and for long wires $k_{F} L \gg 1$

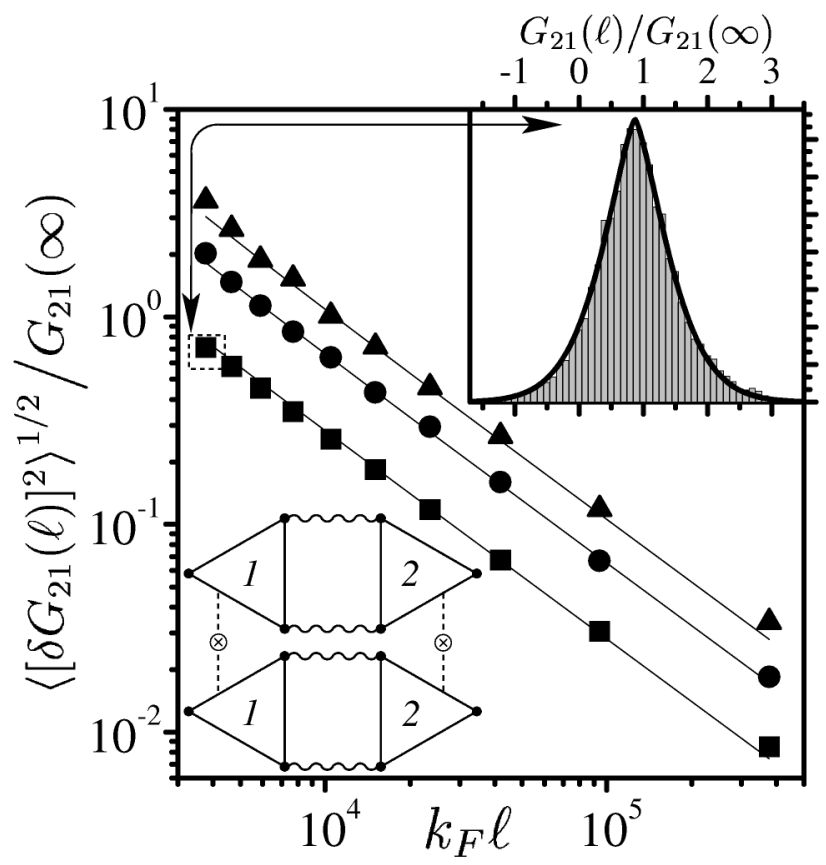

FIG. 2. Relative fluctuations of the transconductance as a function of the mean free path for the following lengths of the two 1D wires: $k_{F} L=100 \pi / 3$ (squares), $200 \pi / 3$ (circles), and $300 \pi / 3$ (triangles). The full lines are the results of Eq. (5) which is shown diagrammatically by the lower left inset. The upper right inset shows a typical histogram based on $10^{4}$ disorder configurations. 
we find

$$
\frac{\left\langle\left[\delta G_{21}(\ell)\right]^{2}\right\rangle^{1 / 2}}{G_{21}(\infty)} \simeq \frac{\left[2\left\langle\mathcal{R}_{1}\right\rangle\left\langle\mathcal{R}_{2}\right\rangle U_{12}^{2}\left(2 k_{F}\right) \tilde{U}_{12}^{2}(0)\right]^{1 / 2}}{U_{12}^{2}\left(2 k_{F}\right)},
$$

where

$$
\begin{aligned}
\tilde{U}_{12}^{2}(0) \equiv & \int_{0}^{L} \int_{0}^{L} \int_{0}^{L} \int_{0}^{L} d x_{1} d x_{2} d x_{1}^{\prime} d x_{2}^{\prime} \\
& \times U_{12}\left(x_{1}, x_{2}\right) U_{12}\left(x_{1}^{\prime}, x_{2}^{\prime}\right) \\
& \times\left(1-\frac{2\left|x_{1}-x_{1}^{\prime}\right|}{L}\right)\left(1-\frac{2\left|x_{2}-x_{2}^{\prime}\right|}{L}\right) .
\end{aligned}
$$

The denominator is the result $G_{21}(\infty) \propto U_{12}^{2}\left(2 k_{F}\right)$ for ballistic wires. For the realistic case where $U_{12}\left(2 k_{F}\right) \ll$ $\widetilde{U}_{12}(0)$ we see that the fluctuations of the drag can exceed the average value. This is in contrast to the fluctuations of the diagonal conductance $\left\langle\left[\delta G_{i i}\right]^{2}\right\rangle^{1 / 2}$, which are vanishing compared to the mean value $\left\langle G_{i i}\right\rangle=\left(2 e^{2} / h\right)(1-$ $\left.\left\langle\mathcal{R}_{i}\right\rangle\right) \sim 2 e^{2} / h$ in the limit of weak disorder. Figure 2 displays the prediction of Eq. (5) along with the numerical results described below and very good agreement is seen.

In order to solve the 1D model numerically, we study Eq. (4) on a lattice using the method of finite differences [10]. The method offers a way of studying disordered systems by ensemble averaging over different disorder configurations [11]. In our numerical example, we use a bare long-ranged Coulomb interaction and the Anderson model with diagonal disorder [12]. We have numerically stud- ied the drag as a function of the mean free path $\ell$ and the length $L$, choosing the Fermi energy corresponding to a quarter-filled band, and for a separation given by $k_{F} d=1$. We calculate $G_{11}, G_{22}$, and $G_{21}$. Since the potentials in the two wires are uncorrelated we in general have $G_{11} \neq G_{22}$, but $\left\langle G_{11}\right\rangle \simeq\left\langle G_{22}\right\rangle$ and $\left\langle\left(\delta G_{11}\right)^{2}\right\rangle \simeq\left\langle\left(\delta G_{22}\right)^{2}\right\rangle$. Our numerical results for distributions, mean values, and fluctuations for $G_{i i}$ are in full agreement with the results of Abrikosov [13]. In the delocalized regime $\ell \gg L$ we find as expected that disorder has almost no effect on $G_{i i}$ and $\left\langle G_{i i}\right\rangle \sim 2 e^{2} / h$ with very small fluctuations. Figure 2 shows $\left\langle\left[\delta G_{21}(\ell)\right]^{2}\right\rangle^{1 / 2}$ normalized by the drag $G_{21}(\infty)$ in the ballistic regime as a function of $k_{F} \ell$. The expected $1 / \ell$ dependence is born out by the numerical calculations, and we also find that the fluctuations increase with the length of the wires. The inset shows a typical histogram of the drag conductance showing that depending on the disorder configuration $G_{21}(\ell)$ can be either higher or lower than in the ballistic regime. Furthermore, note that in agreement with the arguments given above the drag conductance shows a sign reversal for some disorder realizations.

Localized states.-The low temperature expansion Eq. (4), which results in a $T^{2}$ dependence, is valid only if $|\beta\rangle$ belongs to a continuum of states. To investigate the effects due to localized states we split $\Delta$ in two parts, $\Delta=\Delta_{d}+\Delta_{l}$, where the first term is given by Eq. (4), while the second term is due to scattering in and out of localized states,

$$
\begin{array}{r}
\Delta_{l}(x, y ; \omega)=-2 i \pi^{2} \hbar \sum_{\beta \in \text { localized }}\langle x \mid \beta\rangle\langle\beta \mid y\rangle\left\{\phi\left(x, y ; \varepsilon_{\beta}-\hbar \omega\right)\left[n_{F}\left(\varepsilon_{\beta}-\hbar \omega\right)-n_{F}\left(\varepsilon_{\beta}\right)\right]\right. \\
\left.+\phi\left(y, x ; \varepsilon_{\beta}+\hbar \omega\right)\left[n_{F}\left(\varepsilon_{\beta}+\hbar \omega\right)-n_{F}\left(\varepsilon_{\beta}\right)\right]\right\},
\end{array}
$$

where the localized states have been chosen to be real functions, and

$$
\phi(x, y ; \varepsilon)=\sum_{\alpha \gamma} \delta\left(\xi_{\alpha}\right) \delta\left(\xi_{\gamma}\right)\langle\gamma|\hat{I}| \alpha\rangle\langle\alpha \mid x\rangle\langle y \mid \gamma\rangle .
$$

At low temperatures we can approximate $\phi(x, y ; \varepsilon \pm$ $\hbar \omega) \approx \phi\left(x, y ; \varepsilon_{F}\right)$, which allows the temperature dependence to be extracted by integration over $\omega$ in Eq. (1). Furthermore, for temperatures less than the level spacing the response will be dominated by the coupling to the localized level lying closest to the Fermi level. There are thus three different types of contributions corresponding to the response due to localized or delocalized states in each subsystem, $G_{21}=\frac{e^{2}}{h}\left(g_{d-d}+g_{l-d}+g_{l-l}\right)$ where $g_{d-d} \propto T^{2}$. Let us consider, say, $g_{l-d}$ in some detail. We find

$$
\begin{aligned}
g_{l-d} & \propto \hbar \int d \omega \frac{\hbar \omega\left[n_{F}\left(\varepsilon_{1}+\hbar \omega\right)-n_{F}\left(\varepsilon_{1}\right)\right]}{k T \sinh ^{2}(\hbar \omega / 2 k T)} \\
& \simeq \frac{5 k T}{\cosh \left[0.57\left(\varepsilon_{1}-\varepsilon_{\mathrm{F}}\right) / k T\right]},
\end{aligned}
$$

where $\varepsilon_{1}$ is the energy of the localized level lying closest to the Fermi energy. A similar calculation gives

$$
g_{l-l} \propto \frac{1}{\cosh \left[\left(\varepsilon_{1}-\varepsilon_{F}\right) / 2 k T\right] \cosh \left[\left(\varepsilon_{2}-\varepsilon_{F}\right) / 2 k T\right]} .
$$

The relative strengths of these terms can be estimated as

$$
\frac{g_{d-d}}{g_{l-l}} \sim\left(\frac{k T}{\varepsilon_{F}} k_{F} \sqrt{\mathcal{A}}\right)^{2}, \quad \frac{g_{d-d}}{g_{d-l}} \sim\left(\frac{k T}{\varepsilon_{F}} k_{F} \sqrt{\mathcal{A}}\right),
$$

with $\mathcal{A}$ being the interaction area. Thus at low temperature the contributions due to localized states will dominate. The temperature dependence is very different from the usual $T^{2}$ law, and it may even be temperature independent if both $\varepsilon_{1}$ and $\varepsilon_{2}$ lie on the Fermi level. By adjusting the Fermi energy or system parameters, one can use the drag response to probe the properties and statistics of localized states.

Random matrix theory.-We now discuss the statistical properties of the transconductance. This is important in order to determine the size of the Coulomb drag for an ensemble of disordered mesoscopic systems, such as suggested in Fig. 1. Our starting point is the low temperature result (4) (neglecting localized states). For the calculation 
we need the statistical properties of the $S$ matrix, the eigenstates, and the eigenvalues. We assume that the region where the subsystems couple by Coulomb interactions are disordered and that they can be described by random matrix theory [14]. This means that the eigenvalues and the wave functions are assumed to be uncorrelated and furthermore that the current matrix elements $I_{\alpha \beta}$ are uncorrelated with the value of wave functions. The latter follows from the fact that the current matrix elements are independent of position and may be evaluated outside the disordered region, and hence do not correlate with the wave functions inside the disordered region. With these approximations

$$
\begin{aligned}
\frac{\left\langle\Delta\left(\omega, \mathbf{r}, \mathbf{r}^{\prime}\right)\right\rangle}{4 \omega \pi^{2} \hbar^{2}} \simeq & \operatorname{Im} \sum_{\alpha \beta \gamma}\left\langle I_{\alpha \gamma}\right\rangle \\
& \times\left\langle\rho_{\alpha \beta} \rho_{\beta \gamma}^{\prime} \delta\left(\xi_{\alpha}^{F}\right) \delta\left(\xi_{\beta}^{F}\right) \delta\left(\xi_{\gamma}^{F}\right)\right\rangle .
\end{aligned}
$$

The average of the current matrix element is evaluated using standard random matrix theory [14], and both with

and without time reversal symmetry we find $\left\langle I_{\alpha \gamma}\right\rangle=$ $(\hbar / 2 m)\left\langle\left(\tau^{3}+S^{\dagger} \tau^{3} S\right)_{\alpha \gamma}\right\rangle \propto \tau_{\alpha \gamma}^{3}$. Since the second average in $\langle\Delta\rangle$ is symmetric with respect to interchange of $\alpha$ and $\gamma$, we get $\langle\Delta\rangle=0$ and, of course, therefore $\left\langle G_{21}\right\rangle=0$. The fluctuations are, however, nonzero and involve the average $\left\langle\Delta\left(\omega, \mathbf{r}, \mathbf{r}^{\prime}\right) \Delta\left(\tilde{\omega}, \mathbf{s}, \mathbf{s}^{\prime}\right)\right\rangle$ and hence the combination $\left\langle\left(S^{\dagger} \tau^{3} S\right)_{\alpha \beta}\left(S^{\dagger} \tau^{3} S\right)_{\alpha^{\prime} \beta^{\prime}}\right\rangle$, which in the limit of a large $N$ becomes $(2 N)^{-2} \delta_{\alpha \beta^{\prime}} \delta_{\alpha^{\prime} \beta}$. Interestingly, again the result is not changed by breaking of time reversal symmetry, in contrast to the case of universal conductance fluctuations, where the results with or without an applied $B$ field differ by a factor of 2 [14]. The variance of the $\Delta$ then reads

$$
\frac{\left\langle\Delta\left(\omega, \mathbf{r}, \mathbf{r}^{\prime}\right) \Delta\left(\tilde{\omega}, \mathbf{s}, \mathbf{s}^{\prime}\right)\right\rangle}{\pi^{2} \omega \tilde{\omega}} \simeq \frac{C\left(\mathbf{r}, \mathbf{r}^{\prime}, \mathbf{s}^{\prime}, \mathbf{s}\right)-C\left(\mathbf{r}, \mathbf{r}^{\prime}, \mathbf{s}, \mathbf{s}^{\prime}\right)}{(2 N)^{2}}
$$

where $C$ is a correlation function involving four density matrices

$$
\begin{aligned}
C\left(\mathbf{r}, \mathbf{r}^{\prime}, \mathbf{s}, \mathbf{s}^{\prime}\right) & =\sum_{\alpha \alpha^{\prime} \beta \beta^{\prime}}\left\langle\rho_{\alpha \beta}(\mathbf{r}) \rho_{\beta \alpha^{\prime}}\left(\mathbf{r}^{\prime}\right) \rho_{\alpha^{\prime} \beta^{\prime}}(\mathbf{s}) \rho_{\beta^{\prime} \beta}\left(\mathbf{s}^{\prime}\right) \delta\left(\xi_{\alpha}^{F}\right) \delta\left(\xi_{\beta}^{F}\right) \delta\left(\xi_{\alpha^{\prime}}^{F}\right) \delta\left(\xi_{\beta^{\prime}}^{F}\right)\right\rangle \\
& \simeq \frac{1}{(2 \pi)^{4}}\left\langle A\left(\mathbf{r}, \mathbf{r}^{\prime}\right)\right\rangle\left\langle A\left(\mathbf{s}, \mathbf{s}^{\prime}\right)\right\rangle\left\langle A\left(\mathbf{r}, \mathbf{s}^{\prime}\right)\right\rangle\left\langle A\left(\mathbf{r}^{\prime}, \mathbf{s}\right)\right\rangle
\end{aligned}
$$

to lowest order in $1 / k_{F} \ell$. Using the average spectral function relevant to the $2 \mathrm{D}$ case $\langle A(r)\rangle \simeq\left(m / 2 \hbar^{2}\right) \times$ $\exp (-r / 2 \ell) J_{0}\left(k_{F} r\right)$, and assuming, in addition to $k_{F} \ell \gg 1$, also that $\ell \gg r_{s}$, where $r_{s}$ is the screening length, we obtain the estimate

$$
\left\langle\delta G_{21}^{2}\right\rangle^{1 / 2} \approx 10^{-4} \frac{e^{2}}{h}\left(\frac{k T}{\varepsilon_{F}} \frac{U_{12}(d)}{\varepsilon_{F}}\right)^{2} \frac{r_{s}^{2} k_{F} \sqrt{\mathcal{A}}}{\ell^{2} N^{2}} .
$$

With typical numbers for GaAs 2DEG structures the fluctuations of the transresistance are of the order of $0.1 \Omega$, which should be measurable.

In conclusion, we have studied drag in mesoscopic systems and argue that measurement of transconductance provides an interesting new method for investigation of the electronic properties of these systems.

We thank C. W. J. Beenakker and M. Brandbyge for useful discussions.

[1] For a review see A. G. Rojo, J. Phys. Condens. Matter 11, R31 (1999).

[2] B. Y.-K. Hu and K. Flensberg, in Hot Carriers in Semiconductors, edited by K. Hess (Plenum Press, New York, 1996), p. 943; V. L. Gurevich et al., J. Phys. Condens. Matter 10, 2551 (1998); O. Raichev and P. Vasilopoulos, Phys. Rev. B 61, 7511 (2000).
[3] Y. V. Nazarov and D. V. Averin, Phys. Rev. Lett. 81, 653 (1998); K. Flensberg, ibid. 81, 184 (1998); V. V. Ponomarenko and D. V. Averin, ibid. 85, 4928 (2000).

[4] B. N. Narozhny and I. L. Aleiner, Phys. Rev. Lett. 84, 5383 (2000).

[5] We are aware of the following preliminary work: P. Debray et al., Physica (Amsterdam) 6E, 694 (2000); M. Yamamoto et al. (unpublished); A. Kristensen (private communication); J. Nicholls (private communication).

[6] A. M. Martin and M. Büttiker, Phys. Rev. Lett. 84, 3386 (2000); T. A. Shutenko et al., Phys. Rev. B 61, 10366 (2000); I. D'Amico and G. Vignale, Phys. Rev. B 62, 4853 (2000).

[7] Coulomb drag of extended structures and in the presence of localization has been considered previously; see, e.g., E. Shimshoni, Phys. Rev. B 56, 13301 (1997); B. Tanatar and A. K. Das, ibid. 61, 15959 (2000).

[8] A. Kamenev and Y. Oreg, Phys. Rev. B 52, 7516 (1995).

[9] K. Flensberg et al., Phys. Rev. B 52, 14761 (1995).

[10] S. Datta, Electronic Transport in Mesoscopic Systems (Cambridge University Press, Cambridge, 1995).

[11] The disorder in the subsystems can be both uncorrelated [9] or correlated, I. V. Gornyi et al., Phys. Rev. Lett. 83, 152 (1999).

[12] P. W. Anderson, Phys. Rev. 109, 1492 (1958).

[13] A. A. Abrikosov, Solid State Commun. 37, 997 (1981).

[14] C. W. J. Beenakker, Rev. Mod. Phys. 69, 731 (1997).

[15] For the realization of a very similar system see U. Wilhelm and J. Weis, Physica (Amsterdam) 6E, 668 (2000). 